\title{
Guía de Práctica Clínica: Actualización y combinación de las guías de cuidados preventivos de la fuerza de tareas de EE.UU. y Canadá (Cuarta parte)
}

Combination and update of preventive care guidelines of the U.S. and Canadian task forces

Agustín Ciapponị

\section{Resumen}

La Fuerza de Tareas Preventiva de los EE.UU. (sigla en inglés: USPSTF) y la Fuerza de Tareas Canadiense de Cuidados Preventivos de la Salud (sigla en inglés: CTFPHC) son las dos instituciones más importantes del mundo en la evaluación de cuidados preventivos. Ambas emiten y actualizan constantemente recomendaciones preventivas; y las mismas pueden accederse en forma completa y gratuita a través de sus sitios Web (http://www.ahrq.gov/clinic/cps3dix.htm, y http://www.ctfphc.org). En esta cuarta entrega continuamos con la actualización de las prácticas preventivas referidas al rastreo del cáncer y su justificación clínica emitida por ambas entidades, respecto de aquellas previamente resumidas en el $2003^{1}$ y en el $2005 / 6^{2,3}$ en Evidencia. Estas habían alcanzado las recomendaciones generadas hasta septiembre de 2005; lo cual implica la actualización o incorporación de aquellas recomendaciones aquí publicadas con fecha posterior. Muchas recomendaciones emitidas hace varios años se encuentran bajo revisión pero las mantenemos a título informativo hasta que los cambios sean confirmados.

\section{Abstract}

The US Preventive Services Task Force (USPSTF) and the Canadian Task Force on Preventive Health Care (CTFPHC) are the two most important institutions in the world in the evaluation of preventive care. Both released and updated preventive recommendations, which can be accessed in full and free through its Web sites (http://www.ahrq.gov/clinic/cps3dix.htm and http://www.ctfphc.org ). In this fourth issue we continue to update on preventive screening of cancer and its clinical justification issued by both entities. Many recommendations made several years ago are under review but we keep them until the changes are confirmed.

Palabras clave: guía de práctica clínica, rastreo preventivo, cáncer. Key words: clinical practice guideline, preventive screening, cancer.

Ciapponi A. Actualización y combinación de las guías de cuidados preventivos de las fuerzas de tareas de EE.UU. y Canadá (cuarta parte). Evid Act Pract Ambul. 13(1). 20-25. Ene-mar 2010. Las principales recomendaciones referidas a la prevención del cáncer se resumen en la tabla 1.

Tabla 1: Recomendaciones para la prevención del cáncer según la USPSTF, con aportes complementarios de la CTFPHC

\begin{tabular}{|c|c|c|c|c|c|}
\hline Problema & Población diana & Estrategia de rastreo & \multicolumn{2}{|c|}{ Recomendación } & Publicación \\
\hline \multirow{4}{*}{$\begin{array}{l}\text { Cáncer de cuello } \\
\text { uterino }\end{array}$} & Mujeres sexualmente activas 021 años & \multirow[t]{3}{*}{ Papanicolau (Pap) } & & $\mathrm{I} \oplus \oplus \oplus \oplus$ & \multirow{4}{*}{$1 / 2003$} \\
\hline & Mujeres $>65$ años & & $\mathrm{D}$ & $\mathrm{I} \oplus \oplus \oplus \oplus^{\#}$ & \\
\hline & $\begin{array}{l}\text { Mujeres con antecedente de histerectomía total por } \\
\text { enfermedad benigna }\end{array}$ & & $\mathrm{D}$ & $\mathrm{I} \oplus \oplus \oplus \oplus^{\#}$ & \\
\hline & & Nuevas tecnologías & 1 & $-\oplus \oplus \# \mathrm{OO}$ & \\
\hline \multirow{7}{*}{ Cáncer de mama } & Mujeres de 50 a 74 años & \multirow[t]{3}{*}{ Mamografía } & $B^{*}$ & $\mathrm{II} \oplus \oplus \oplus \mathrm{O}$ & \multirow{7}{*}{$11 / 2009$} \\
\hline & Mujeres de 40 a 49 años & & C & $-\oplus \oplus \# \mathrm{OO}$ & \\
\hline & Mujeres mayores de 75 años & & 1 & $-\oplus \oplus \# \mathrm{OO}$ & \\
\hline & \multirow[t]{4}{*}{ Mujeres mayores de 40 años } & \multirow{2}{*}{$\begin{array}{l}\text { Examen clínico mamario } \\
\text { complementario a la mamografía }\end{array}$} & $\mathrm{I}$ & $-\oplus \oplus \# \mathrm{OO}$ & \\
\hline & & & \multirow{2}{*}{\multicolumn{2}{|c|}{$-\oplus \oplus \# \mathrm{OO}$}} & \\
\hline & & $\begin{array}{l}\text { Mamografía digital, Resonancia } \\
\text { magnética }\end{array}$ & & & \\
\hline & & Auto-examen mamario & $\mathrm{D}$ & $\mathrm{I} \oplus \oplus \oplus \mathrm{O}$ & \\
\hline \multirow{2}{*}{$\begin{array}{l}\text { Cáncer de mama y } \\
\text { ovario }\end{array}$} & Mujeres con riesgo elevado según la historia familiar & \multirow{2}{*}{$\begin{array}{l}\text { Consejo genético y búsqueda de } \\
\text { mutación del gen BRCA }\end{array}$} & B & II $\oplus \oplus \oplus \oplus$ & \multirow[t]{2}{*}{$9 / 2005$} \\
\hline & Mujeres con riesgo promedio según la historia familiar & & D & $\mathrm{I} \oplus \oplus \oplus \oplus^{*}$ & \\
\hline Cáncer de ovario & Mujeres con riesgo habitual & CA-125 o ecografía transvaginal & $\mathrm{D}$ & $\mathrm{I} \oplus \oplus \oplus \oplus^{\#}$ & $5 / 2004$ \\
\hline \multirow[t]{2}{*}{ Cáncer prostático } & Hombres de 50 a 74 años & \multirow[t]{2}{*}{ PSA } & $\mathrm{I}$ & $\mathrm{I} \oplus \oplus \mathrm{OO}$ & \multirow[b]{2}{*}{$8 / 2008$} \\
\hline & Hombres 75 años & & D & $\mathrm{I} \oplus \oplus \oplus \mathrm{O}$ & \\
\hline \multirow[t]{4}{*}{ Cáncer colorectal } & Adultos de 50 a 75 años & $\begin{array}{c}\text { SOMF, rectosigmoidoscopía } 0 \\
\text { colonoscopía }\end{array}$ & $A^{*}$ & $\mathrm{I} \oplus \oplus \oplus \oplus$ & \multirow{4}{*}{$10 / 2008$} \\
\hline & & Colonoscopía virtual o ADN fecal & 1 & $-\oplus \oplus \# \mathrm{OO}$ & \\
\hline & Adultos de 76 a 85 años & & C & $-\oplus \oplus \oplus \mathrm{O}$ & \\
\hline & Adultos de más de 85 años & & $\mathrm{D}$ & $\mathrm{I} \oplus \oplus \oplus \mathrm{O}$ & \\
\hline Cáncer vesical & Adultos & Tiras reactivas, citología & $D^{*}$ & $\mathrm{I} \oplus \oplus \oplus \oplus^{\#}$ & $6 / 2004$ \\
\hline Cáncer de pulmón & Adultos & $\begin{array}{c}\text { Tomografía computada de baja dosis, } \\
\text { radiografía de tórax o citología de } \\
\text { esputo }\end{array}$ & $\mathrm{I}^{*}$ & $-\oplus \oplus \# \mathrm{OO}$ & $5 / 2004$ \\
\hline Cáncer oral & Adultos & Examen físico & $\mathrm{I}$ & $-\oplus \oplus \# \mathrm{OO}$ & 2/2004 \\
\hline Cáncer de páncreas & Adultos & $\begin{array}{c}\text { Palpación abdominal, ecografía } 0 \\
\text { marcadores serológicos }\end{array}$ & $\mathrm{D}$ & $\mathrm{I} \oplus \oplus \oplus \oplus^{*}$ & $2 / 2004$ \\
\hline Cáncer de testículo & Adolescentes y adultos & Examen físico 0 auto-examen & D & $\mathrm{I} \oplus \oplus \oplus \oplus^{\#}$ & $2 / 2004$ \\
\hline Cáncer de Piel & Adultos & Examen corporal total 0 auto-examen & $\mathrm{I}^{\star}$ & $-\oplus \oplus \mathrm{AOO}$ & $2 / 2009$ \\
\hline \multirow[t]{2}{*}{ Cáncer de tiroides } & Población general (niños 0 adultos) & & $\mathrm{D}$ & $\mathrm{I} \oplus \oplus \oplus \oplus^{\#}$ & 1996 \\
\hline & $\begin{array}{l}\text { Personas con historia de irradiación de cabeza/cuello en la } \\
\text { infancia }\end{array}$ & & 1 & $-\oplus \oplus \# \mathrm{OO}$ & \\
\hline
\end{tabular}

SOMF: sangre oculta en materia fecal. Un * sobre el grado de recomendación indica que ésta puede modificarse bajo ciertas circunstancias explicadas en el apartado correspondiente o por observaciones hechas por la CTFPHC. Grados de Evidencia: Buena (GRADE Alta $\oplus \oplus \oplus \oplus$ ), Aceptable (GRADE Moderada $\oplus \oplus \oplus)$ e Insuficiente $($ GRADE Baja $\oplus \oplus$ OO / Muy Baja $\oplus$ OOO). El \# indica que algunos componentes de la recomendación están basados también en un nivel inferior de evidencia. Por ejemplo $\oplus \oplus \#$ OO significa que una recomendación está basada en niveles $\oplus$ OOO y $\oplus \oplus$ OO. Nivel de recomendación: A Recomienda fuertemente el servicio (GRADE I: Fuerte); B: Recomienda el servicio (GRADE II: Débil); C Recomienda no proveer el servicio de manera rutinaria (GRADE no se expide); D: Recomienda no proveer el servicio (GRADE I: Fuerte); I: No puede hacerse una recomendación por evidencia insuficiente (GRADE no se expide). 
Cáncer de cuello uterino, en mujeres sexualmente activas $0 \geq 21$ años (A)

Se encontró buena evidencia, proveniente de múltiples estudios observacionales, de que el rastreo mediante la citología cervical (Pap con espátula y cepillo) reduce la incidencia y mortalidad del cáncer cervical. Sin embargo, la evidencia directa resulta limitada para determinar el intervalo y la edad óptima de comienzo y finalización del rastreo. La evidencia indirecta sugiere que la mayoría de la ventajas pueden obtenerse comenzando el rastreo dentro de los tres años del inicio de la actividad sexual o a la edad de 21 años (cualquiera que se alcance primero, y dependiendo de la precocidad del debut sexual poblacional la edad podría ser menor), a intervalos de por lo menos cada tres años.

Dado que la sensibilidad de un solo Pap para detectar lesiones de alto grado es del 60 a $80 \%$, la mayoría de las organizaciones recomiendan realizar el Pap anualmente hasta que al menos dos o tres Paps hayan sido normales. Recién entonces podría prolongarse el intervalo de rastreo.

Los factores de riesgo que justifican el rastreo anual incluyen el comportamiento sexual de riesgo, y la presencia de alguno de los siguientes antecedentes personales: neoplasia cervical, infección por HPV u otras enfermedades de transmisión sexual (ETS); aunque la evidencia resulta limitada como para determinar los beneficios de esta estrategia.

Se concluye que los beneficios del rastreo superan ampliamente los daños potenciales.

Cáncer de cuello uterino, en mujeres de 65 años o mayores (D) La evidencia resulta limitada para determinar los beneficios de continuar el rastreo en mujeres mayores de 65 años. Además, en las mujeres de este grupo que ya han sido previamente rastreadas, el rédito diagnostico es bajo debido a la incidencia declinante de lesiones cervicales de alto grado.

Por otro lado, existe buena evidencia de el rastreo de mujeres $>65$ años está asociado a un riesgo creciente de daño, incluyendo la obtención de resultados falso-positivos y la realización de procedimientos invasivos innecesarios. Por ello no se recomienda rastrear rutinariamente a las mujeres mayores de 65 años que han tenido Paps recientes adecuados y que no presentan un riesgo elevado de cáncer cervical por otras causas.

Las nuevas recomendaciones de la Sociedad Americana del Cáncer (sigla en inglés ACS) sugieren suspender el rastreo a los 70 años siempre que se cuenten con al menos tres Paps consecutivos previos técnicamente satisfactorios y documentados como normales o negativos, y que no existan Paps anormales en los últimos diez años. En mujeres mayores de esta edad el rastreo podría efectuarse si las mismas no hubieran sido rastreadas previamente, cuando la información histórica sobre el rastreo fuese inasequible o cuando resulte poco probable que el rastreo haya ocurrido en el pasado (en sitios sin programas de rastreo).

Se concluye que el daño potencial del rastreo probablemente exceda a los beneficios en las mujeres mayores de 65 años que han tenido previamente resultados normales y que no presentan un riesgo elevado de cáncer cervical.

Cáncer de cuello uterino, en mujeres con antecedente de histerectomía total por enfermedad benigna (D)

Se encontró buena evidencia de que el rédito diagnóstico del rastreo citológico es muy bajo en mujeres histerectomizadas, y evidencia pobre de que el rastreo para detectar el cáncer vaginal mejore los resultados de salud. Sin embargo, debe certifi- carse la ausencia de cérvix mediante la lectura del parte quirúrgico o mediante la realización de una especuloscopía.

Por otro lado, si bien son escasos los datos sobre rédito diagnóstico de esta estrategia de rastreo, en mujeres con historia del cáncer cervical invasor o con antecedentes de exposición al Dietilestilbestrol, el rastreo deberá continuarse debido al incremento de riesgo de cáncer vaginal.

Se concluye que el daño potencial de continuar el rastreo luego de una histerectomía probablemente exceda a los beneficios.

Cáncer de cuello uterino, mediante el uso de nuevas tecnologías (I)

La evidencia encontrada resulta pobre para determinar si nuevas tecnologías, tales como la citología en medio líquido (ThinPrep ${ }^{\circledast}$, AutoCyte PREP ${ }^{\circledR}$ ), el re-rastreo computarizado (PapNet ${ }^{\circledR}$ ), o el rastreo basado en algoritmos (AutoPap ${ }^{\circledR}$ ) son más eficaces que el rastreo convencional, mediante Pap, en la reducción de la incidencia o mortalidad del cáncer cervical invasor. La evidencia que determina la sensibilidad y la especificidad de estas tecnologías de rastreo mas novedosas también resulta limitada. Incluso de resultar mejores en términos de sensibilidad, los estudios que modelaron el uso de tales tecnologías sugieren que estas no serían rentables a menos que sean utilizados con intervalos mayores o iguales a tres años.

Tampoco se encontró evidencia suficiente como para determinar el balance entre los beneficios o daños potenciales del rastreo de HPV adjunto o como alternativa al rastreo por Pap. Los ensayos en curso deberán clarificar pronto el papel de las pruebas de HPV en el rastreo del cáncer cervical.

Consecuentemente, se concluye que no puede determinarse si los beneficios potenciales al uso de estas nuevas técnicas de rastreo son suficientes como para justificar un aumento posible de los daños o costos asociados.

Cáncer de mama, en mujeres de 50 a 74 años mediante mamografía (B)

Se encontró evidencia convincente de que la mamografía realizada en forma periódica reduce la mortalidad por cáncer de mama. Esta reducción absoluta resulta mayor para el grupo etáreo comprendido entre los 50 a 74 años comparado con el grupo de 40 a 49. La evidencia a su vez es más fuerte para las mujeres de 60-69 años. En las mujeres mayores de 75 años la evidencia resulta escasa.

Los daños resultantes del rastreo del cáncer de mama incluyen los de índole psicológica, la realización innecesaria de imágenes en mujeres sin cáncer, y los inconvenientes asociados a los diagnósticos falso-positivos (biopsias y tratamientos innecesarios). También tiene que tenerse en cuenta la posibilidad de sobrediagnóstico y sobretratamiento (véase comentario editorial).

En los ensayos clínicos que se demostró reducción de la mortalidad, los intervalos de rastreo con mamografía fueron de 12 a 33 meses. Sin embargo la adopción de un intervalo bianual sobre el rastreo mamográfico anual mantiene la mayoría de estos beneficios, reduciendo casi a la mitad la posibilidad de daños.

Por lo expuesto se concluye que existe moderada certeza de un beneficio moderado en el rastreo bianual mediante mamografía en las mujeres de 50 a 74 años.

La CTFPHC recomienda tanto la mamografía como el examen clínico en las mujeres de 50 a 69 años, con intervalos de uno a dos años (A). 
Cáncer de mama, en mujeres de 40 a 49 años mediante mamografía (C)

Los resultados falso-positivos y sus consecuencias potenciales son mas frecuentes en las mujeres de 40 a 49 años. Además, el número necesario a rastrear (para salvar una vida) resulta más elevado que en el grupo de 50 a 74 años. Por ello se concluye que existe evidencia suficiente de que el beneficio neto del rastreo es menor en este grupo de mujeres.

Se recomienda entonces considerar el contexto personal y los deseos de las mujeres menores de 50 años, así como discutir la ecuación riesgo beneficio para decidir a favor o en contra de iniciar el rastreo bianual mediante mamografía.

Cáncer de mama, en mujeres mayores de 75 años mediante mamografía (I)

Los ensayos clínicos sobre rastreo de cáncer de mama no han incluido mujeres de este grupo etáreo. Si bien el cáncer de mama es una de las principales causas de muerte en las mujeres mayores, también es cierto que el sobrediagnóstico y sobretratamiento de estas lesiones adquiere una dimensión mayor en este grupo de mujeres.

Se concluye que la evidencia es insuficiente para valorar los beneficios adicionales y los daños del rastreo mediante mamografía en las mujeres de 75 años o mayores.

Cáncer de mama, en mujeres mayores de 40 años mediante el examen clínico mamario complementario al rastreo por mamografía (I)

Ningún ensayo clínico examinó los beneficios del examen mamario exclusivo (sin mamografía) comparado a ningún rastreo. Por ende, no se pudieron determinar los beneficios del exa-men mamario exclusivo o la ventaja incremental de agregar el mismo a la estrategia de rastreo mediante mamografía.

Cáncer de mama, mediante mamografía digital o resonancia magnética nuclear (I)

La evidencia sobre el uso de estas tecnologías en el rastreo del cáncer de mama resulta insuficiente. Por lo tanto no puede establecerse aun el balance entre los beneficios y los daños.

Cáncer de mama, mediante el auto-examen mamario (D) Se encontró evidencia suficiente que sugiere que recomendar y enseñar el auto-examen mamario no reduce la mortalidad por cáncer de mama. Asimismo, se encontró evidencia aceptable de que el auto-examen mamario está asociado a un riesgo creciente de resultados falso-positivos, lo cual deriva en ansiedad, aumento de las visitas medicas, imágenes adicionales y biopsias. Se concluye entonces que los daños superan los beneficios.

Cáncer de mama y ovario, en mujeres con alto riesgo según su historia familiar mediante consejo genético y búsqueda de mutación del gen BRCA (B)

Se encontró evidencia aceptable de que las mujeres con ciertos patrones familiares específicos poseen un riesgo incrementado de desarrollar cáncer de ovario o mama asociado a la mutación genética BRCA1 o BRCA2.

Se considera historia familiar de riesgo a la presencia de: dos familiares de primer grado con cáncer mamario (uno de éstos con diagnóstico antes de los 50 años); tres o mas familiares de primer o segundo grado con cáncer mamario, independientemente de la edad al diagnóstico; la presencia de familiares de primer o segundo grado con cáncer mamario y ovárico; un familiar de primer grado con cáncer mamario bilateral; dos o mas familiares de primer o segundo grado con cáncer ovárico, independientemente de la edad al diagnóstico; un familiar de primer o segundo grado con cáncer mamario y ovárico; y el antecedente de cáncer mamario en un familiar varón.

Estas mujeres se beneficiarían al recibir consejo genético efectuado por personal entrenado, que permita tomar una decisión informada sobre la realización de la prueba genética y de los posibles tratamientos profilácticos.

Existe sin embargo evidencia insuficiente de que la quimioprofilaxis o la realización de rastreos intensivos mejoren los resultados en salud de las mujeres BCRA+, aunque hay bastante evidencia de que la cirugía profiláctica reduce la incidencia de cáncer de mama u ovario. La evidencia resulta insuficiente sobre las consecuencias éticas, legales y sociales de derivar a consejo genético, seguido de la realización de la prueba específica; pero los riesgo de la cirugía profiláctica son conocidos.

Se concluye que los beneficios de derivar a mujeres con historia familiar de riesgo para un consejo genético provisto por personal capacitado superan los daños potenciales

Cáncer de mama y ovario, en mujeres con riesgo promedio según la historia familiar mediante consejo genético y búsqueda de mutación del gen BRCA (D)

Se encontró evidencia aceptable de que las mujeres sin los patrones previamente descriptos de historia familiar de cáncer de mama u ovario tienen un riesgo bajo de desarrollar cáncer de mama u ovario asociado a la mutación genética BRCA1 o BRCA2, por lo que los beneficios del rastreo de esta ultima condición serían nulos o casi nulos.

Considerando los riesgos previamente mencionados se concluyó que los daños superan a los beneficios potenciales.

Cáncer de ovario, en mujeres mediante $\mathrm{CA}-125$ sérico o ecografía transvaginal (D)

Se encontró evidencia aceptable de que el rastreo con antígeno CA-125 sérico o ecografía transvaginal puede descubrir el cáncer ovárico en una fase más temprana; sin embargo, también existe evidencia aceptable de que la detección temprana tendría un efecto probablemente pequeño, en el mejor de los casos, sobre la reducción de la mortalidad específica.

Debido a la baja prevalencia de cáncer ovárico y la naturaleza invasiva del diagnóstico después de una prueba de rastreo positiva, hay evidencia aceptable de que el rastreo podría llevar a daños importantes.

Se concluye que los daños superan a los beneficios potenciales.

Cáncer prostático, en hombres de 50 a 74 años mediante PSA cada uno a cuatro años (I)

El antígeno de prostático específico (sigla en ingles, PSA) resulta más sensible que el tacto rectal para la detección del cáncer prostático. Si bien el punto de corte convencionalmente utilizado de 4,0 $\mathrm{g} / \mathrm{L}$ reduce su sensibilidad para detectar cánceres tempranos, un valor menor aumentaría significativamente la tasa de resultados falso-positivos.

Las variaciones del rastreo con PSA no han demostrado mejorar los resultados de salud. Se encontró buena evidencia de que el rastreo con PSA puede detectar algunos casos de cáncer prostático, pero la evidencia resulta inconsistente y poco concluyente en demostrar que la detección temprana 
mejore resultados de salud en comparación con el tratamiento efectuado a partir de la detección clínica.

Por otro lado, el rastreo se asocia a daños de moderados a importantes incluyendo resultados falso-positivos, ansiedad innecesaria, biopsias frecuentes (con su consecuente malestar asociado), y las complicaciones potenciales inherentes al tratamiento, como disfunción eréctil, incontinencia urinaria, disfunción intestinal o la muerte. Estos daños son especialmente importantes debido a que algunos hombres tratados por cáncer de próstata, nunca presentarían síntomas relacionados con el cáncer durante su vida.

Por lo expuesto, se concluye que la evidencia resulta insuficiente para determinar si los beneficios compensan los daños. Si la detección mediante PSA redujera la mortalidad, el rastreo cada cuatro años sería equivalente a la anual. Los médicos deben discutir con sus pacientes los beneficios potenciales y los daños conocidos de la detección mediante PSA. Los hombres en este grupo de edad deben ser informados de las brechas de la evidencia y sus preferencias personales deben guiar la decisión de si desea ordenar el examen.

\section{Cáncer prostático, en hombres $\geq 75$ años (D)}

En los hombres de 75 años o más años de edad (o también en aquellos con menos de diez años de expectativa de vida) se encontró evidencia suficiente de que el beneficio relacionado al tratamiento del cáncer de próstata detectado por rastreo es pequeño a nulo.

Se concluye que en este grupo etáreo existe moderada evidencia de que los daños del rastreo superan a los beneficios potenciales.

Cáncer colorectal (CCR), en adultos de 50 a 75 años mediante SOMF, rectosigmoidoscopía o colonoscopía (A) Se encontró evidencia de moderada a buena que la prueba de sangre oculta en materia fecal (SOMF) y la rectosigmoidoscopía, sola o conjuntamente con SOMF, reducen la mortalidad por CCR.

No se encontró evidencia directa de que el rastreo mediante colonoscopía sea eficaz en reducir la mortalidad por CCR. La eficacia de la colonoscopía se sustenta por su papel integral en ensayos de SOMF, de la extrapolación de estudios que evaluaron la rectosigmoidoscopía, de evidencia limitada proveniente de estudios caso-control, y de la capacidad de la colonoscopía de examinar el colon proximal.

Aunque la colonoscopía es considerada el estudio de referencia contra la que se comparan otras pruebas, su sensibilidad no es perfecta. Dos tipos de estudios que comparaban la colonoscopía y la colonoscopía virtual mostraron que la colonoscopía pueden perder incluso los pólipos $>10 \mathrm{~mm}$ y el CCR. Además, la mayoría de la evidencia sobre la sensibilidad de la colonoscopía proviene de examinadores con experiencia superior a la de aquellos que se desempeñan en atención primaria.

El colon por enema doble contraste es una alternativa para examinar la totalidad del colon, pero es menos sensible que la colonoscopía, y no hay evidencia directa de su eficacia en la reducción de la mortalidad.

Existe evidencia de aceptable a buena que la selección de cualquiera de las tres pruebas recomendadas reduce la mortalidad del CCR en adultos de 50 a 75 años. El seguimiento de los resultados positivos no colonoscópicos requiere la confirmación por colonoscopía con independencia de la prueba utilizada. El principal daño en el cribado del CCR se debe a la utilización inicial de procedimientos invasivos o a su uso secuencial. Los daños pueden surgir de la preparación del paciente, de la sedación utilizada durante el procedimiento, y del propio procedimiento.

Existe suficiente evidencia para estimar los daños atribuibles a la colonoscopía. La perforación colónica ocurre en 3,8 por cada 10.000 procedimientos. Las complicaciones más serias (muertes atribuibles a la colonoscopía o reacciones adversas que requieran internación, incluyendo perforación, sangrado mayor, diverticulitis, dolor abdominal severo y eventos cardiovasculares) son aun más frecuentes y ocurren en aproximadamente 25 por cada 10.000 procedimientos.

En cuanto a la rectosigmoidoscopía, existe suficiente evidencia de que se producen complicaciones graves en aproximadamente 3,4 por cada 10.000 procedimientos. No hay evidencia acerca de los daños de la SOMF, pero se estiman como pequeños. El principal beneficio de las pruebas menos invasivas es que pueden reducir el número necesario de colonoscopias y sus riesgos.

Se concluye que existe buena evidencia de que los beneficios de rastrear adultos a partir de los 50 años y hasta los a 75 años mediante SOMF anual, rectosigmoidoscopía cada cinco años (combinada con SOMF cada tres años) o colonoscopía cada 10 años compensan substancialmente los daños potenciales.

A pesar de las diferentes alternativas de rastreo, los datos disponibles resultan insuficientes para determinar que estrategia es la mejor en términos del balance de riesgos, beneficios o costo-efectividad. Los modelos matemáticos sugieren que el número de años de vida ganados sería mayor con las pruebas de mayor sensibilidad. A su vez, en todas las modalidades de rastreo una mala adherencia disminuye sustancialmente la eficacia; y a nivel individual, la adherencia a un régimen de rastreo será más importante en los años de vida ganado que el régimen seleccionado. Sin embargo, los datos actuales son insuficientes como para predecir la adhesión a algún régimen de rastreo a nivel poblacional.

Estas recomendaciones se aplican también a las personas con familiares de primer grado que han tenido adenomas o CCR. Sin embargo resulta razonable anticipar el inicio del rastreo a quienes tienen familiares de primer grado que desarrollaron cáncer a una edad más temprana o quienes tengan múltiples parientes de primer grado con CCR.

Estas recomendaciones no se aplican a las personas con síndromes hereditarios específicos (síndrome de Lynch o poliposis adenomatosa familiar) y aquellos con enfermedad inflamatoria intestinal.

Según la CTFPHC, en individuos mayores de 50 años con riesgo habitual de CCR, existe buena evidencia para incluir en forma anual o bianual la prueba de SOMF (A), y evidencia suficiente para incluir a la rectosigmoidoscopía (B) en el rastreo periódico de CCR. Sin embargo la evidencia resulta insuficiente para recomendar solo una o ambas pruebas en forma conjunta (C) o para recomendar o no el uso de la colonoscopía como prueba inicial de rastreo (C).

Existe evidencia suficiente para incluir desde la pubertad el rastreo genético de CCR en los individuos con antecedentes de poliposis adenomatosa familiar, seguido de rectosigmoidoscopía en aquellos individuos con pre-sencia de la mutación genética (B). Los individuos que provienen de familias dónde la mutación del gen se hubiera identificado en algún integrante pero que resultan negativos para dicha prueba solamente requieren rastreo similar a población de riesgo promedio. Los individuos de riesgo dónde la mutación no se haya identificado en la familia o donde el estudio genético no este disponible, 
requieren rastreo semestral o anual comenzando desde la pubertad mediante una rectosigmoidoscopía. En todos los casos debe realizarse el consejo genético antes del estudio genético.

También existe evidencia suficiente para incluir el rastreo de CCR en los familiares de individuos con cáncer de colon hereditario sin poliposis, también conocido como síndrome de Lynch (B). Según los criterios de Ámsterdam para sospechar este síndrome deben estar afectados tres familiares, de los cuales dos deben pertenecer a generaciones sucesivas $\mathrm{y}$ al menos uno de ellos debe tener menos de 45 años al diagnóstico. En estos casos la incidencia de cáncer de colon derecho se halla incrementada y por ello se recomiendo el rastreo mediante colonoscopía en vez de rectosigmoidoscopía. En este grupo resultan inciertas tanto las edades de comienzo como la frecuencia con que debe realizarse la colonoscopía.

Por otro lado existe evidencia insuficiente para recomendar la colonoscopía en pacientes con antecedentes familiares de pólipos colónicos o cáncer que no cumplen con los criterios de las dos entidades previamente señaladas (C).

Cáncer colorectal, en adultos de 50 a 75 años mediante colonoscopía virtual o ADN fecal (I)

La evidencia resulta aun insuficiente para demostrar que la colonoscopía por tomografía computada, llamada colonoscopía virtual (CV), mejore los resultados de salud. Hasta un $16 \%$ de las personas sometidas a su primer CV presentan anomalías extra-colónicas que requieran la realización de nuevos estudios. Aunque la evidencia resulta insuficiente como para evaluar las consecuencias clínicas de la identificación de estas anomalías, podrían desprenderse daños y beneficios potenciales. Entre los primeros se incluyen los relacionados a la realización de pruebas diagnósticas adicionales y procedimientos de las lesiones encontradas, los cuales podrían no tener importancia clínica. Esto impacta tanto sobre el paciente como el sistema de salud.

Los riesgos asociados con la perforación por CV se estiman en 0 a 6 por 10.000 procedimientos. Sin embargo, estas estimaciones podrían ser más elevadas que lo esperable exclusivamente por el rastreo, dado que los estudios de donde provienen incluyeron poblaciones sintomáticas. La exposición acumulada a la radiación por CV es otro problema potencial a considerar. Cada exposición a una CV genera una radiación de $10 \mathrm{mSv}$. Los daños de la radiación a esta dosis son inciertos, pero modelos lineales sin umbral predicen que el $1 \%$ o de los expuestos desarrollará cáncer en su vida. Por otro lado, las mejoras en la tecnología de esta práctica redujeron la dosis de radiación.

En cuanto a la utilidad de las pruebas de cribado mediante ADN fecal, no existe evidencia suficiente sobre su sensibilidad y especificidad.

Se concluye que la evidencia resulta insuficiente como para valorar el balance entre los beneficios y daños de la $C V$ y el ADN fecal como modalidades de rastreo de CCR.

\section{Cáncer colorectal, en adultos de 76 a 85 años (C)}

Existe evidencia suficiente que demuestra que los beneficios de la detección e intervención temprana del CCR disminuyen después de los 75 años. Esto se justifica por la presencia de un tiempo de retraso habitual entre el diagnostico por rastreo y la aparición de síntomas y la concurrencia de otras causas de mortalidad.

Se concluye que a esta edad existe moderada certeza de que es los beneficios netos de la detección son pequeños.
Cáncer colorectal, en adultos de más de 85 años (D)

Para esta edad existe moderada certeza de que los beneficios del rastreo no compensan los daños.

Cáncer vesical, en adultos (D)

Existe evidencia aceptable de que el rastreo con las pruebas disponibles (tiras reactivas, examen citológico, y otras tecnologías mas modernas) puede detectar el cáncer vesical en individuos asintomáticos. Sin embargo, el beneficio potencial del rastreo sería pequeño, en el mejor de los casos, por las siguientes razones: existe evidencia aceptable de que muchos de los cánceres descubiertos por rastreo tienen una baja tendencia a progresar a enfermedad invasora; hay una relativa baja prevalencia global de cáncer vesical generador de consecuencias clínicas importantes y hay evidencia limitada de que el tratamiento temprano del cáncer vesical descubierto por rastreo mejore los resultados de salud a largo plazo.

Los daños potenciales de rastrear son al menos pequeños: las pruebas tienen un bajo valor predictivo positivo y generan muchos resultados falso-positivos, que conducen a procedimientos invasivos innecesarios.

Se concluye que los daños potenciales de rastrear el cáncer vesical superan cualquier beneficio potencial.

Según la CTFPHC: existe evidencia suficiente para excluir esta practica del control de salud de la población general (D); mientras que la evidencia resulta insuficiente para rastrear a los individuos de riesgo, es decir hombres $>60$ años, fumadores o ex-fumadores o con exposición laboral a aminas aromáticas (Ej: anilinas) (C).

Cáncer de pulmón, mediante tomografía computada de baja dosis (TCBD), radiografía de tórax, citología de esputo o su combinación (I)

Se encontró evidencia aceptable de que el rastreo de cáncer de pulmón mediante TCBD, radiografía de tórax o citología de esputo puede detectar el cáncer pulmonar en una fase más temprana de lo que se descubriría en una población no rastreada. Sin embargo, la evidencia es pobre en demostrar que cualquiera de estas estrategias disminuya la mortalidad.

Debido a la naturaleza invasiva del diagnóstico y la posibilidad de un alto número de resultados falso-positivos en ciertas poblaciones, existe un riesgo potencial de producir daños significativos con el rastreo.

Se concluye que no puede determinarse el equilibrio entre los beneficios y daños del rastreo del cáncer de pulmón.

Según la CTFPHC no existe evidencia suficiente para expedirse en relación al rastreo del cáncer de pulmón en la población general mediante el uso de tomografía computada espiralada (I). Por otro lado, existe evidencia suficiente para expedirse en contra del rastreo mediante radiografía de tórax y citología de esputo (D).

\section{Cáncer oral, en adultos (I)}

No se encontró evidencia nueva de buena calidad que demuestre que el rastreo del cáncer oral mejore los resultados en salud, o produzca daños, en los adultos de la población general, y en aquellos con riesgo aumentado (Ej. fumadores de más de 50 años de edad). Por otro lado, resulta improbable que alguna vez se realicen ensayos controlados de rastreo en la población general debido a la muy baja incidencia de esta entidad.

Se concluye que no puede determinarse el equilibrio entre los beneficios y daños de rastrear el cáncer oral. 
Cáncer de páncreas, en adultos asintomáticos mediante palpación abdominal, ecografía o marcadores serológicos (D) No se encontró evidencia alguna de que rastrear el cáncer pancreático sea eficaz para reducir la mortalidad.

Por otro lado, la posibilidad de daño relacionado al rastreo es significativa debido a la prevalencia muy baja de esta entidad, a la exactitud limitada de pruebas de rastreo disponibles, la naturaleza invasiva del diagnóstico luego de realizar una prueba de rastreo positiva, y a los pobres resultados del tratamiento. Se concluye que los daños de rastrear el cáncer pancreático exceden cualquier beneficio potencial.

Cáncer de testículo, en adolescentes y adultos asintomáti$\cos (\mathrm{D})$

No se encontró ninguna evidencia nueva de que el rastreo mediante el examen clínico o el auto-examen testicular sea eficaz para reducir la mortalidad por cáncer testicular. Incluso en ausencia de rastreo, el tratamiento actual proporciona resultados de salud muy favorables.

Dada la baja prevalencia de esta entidad, la exactitud limitada de las pruebas de rastreo y la falta de evidencia de beneficios incrementales del rastreo, se concluyó que los daños exceden cualquier beneficio potencial.

Cáncer de piel, realizado por médicos de atención primaria o auto-examen (I)

Existe evidencia aceptable de que la inspección corporal total realizada por médicos de atención primaria es moderadamente precisa en la detección de melanoma. Sin embargo, la evidencia resulta insuficiente para determinar si esta precisión diagnóstica difiere de la lograda por los dermatógolos, y para determinar cual es la precisión del auto-examen.

Por otro lado, la evidencia también resulta insuficiente para determinar si la detección precoz de cáncer de piel reduce la mortalidad o la morbilidad por esta causa.
Los daños potenciales del rastreo de cáncer de piel incluyen la realización de un diagnóstico equivocado, el sobrediagnóstico y los daños resultantes de biopsias y del sobretratamiento. Se concluye que la evidencia actual resulta insuficiente para evaluar el equilibrio entre beneficios y daños del rastreo de cáncer de piel (carcinoma espinocelular, basocelular y melanoma) realizado por médicos de atención primaria o mediante el auto-examen. Esta recomendación se refiere a población general; y si bien los beneficios del rastreo no están probados ni siquiera en pacientes de alto riesgo, la USPSTF no examinó los resultados de la vigilancia sobre pacientes con síndromes familiares como los de nevos atípicos o melanoma (FAM-M). Debido al riesgo aumentado de melanoma, los médicos deberían estar alertas en los pacientes mayores de 65 años, en aquellos con lunares atípicos o con más de 50 lunares. Asimismo los médicos deberían estar alertas en el contexto de un examen físico realizado por otras razones al hallazgo de lesiones con características sospechosas de malignidad (regla del "A", "B", "C", "D"): Asimetría, Bordes irregulares, Coloración heterogénea y Diámetro $>6 \mathrm{~mm}$, las cuales son merecedoras de biopsia.

Según la CTFPHC existe evidencia suficiente para incluir la inspección corporal total en individuos de alto riesgo (por ej. A los familiares de primer grado con melanoma) (B).

Cáncer de tiroides, en niños o adultos (D) El rastreo de cáncer de tiroides mediante palpación o ecografía en niños o adultos no está recomendado.

Cáncer de tiroides, en personas con historia de irradiación de cabeza/cuello en la infancia (I)

La evidencia resulta insuficiente para expedirse a favor o en contra del rastreo de cáncer de tiroides en personas con historia de irradiación de cabeza y cuello en la infancia pero podría recomendarse en base a otras razones.

Recibido el 20/05/2009 y aceptado el 20/06/2009

\section{Referencias}

1. Ciapponi A. Guía de Práctica Clínica Actualizaciones de cuidados preventivos de la USPSTF (3 ra edición, 2000-2003) Disponible en: http://www.foroaps.org/hitalba-pagina-articulo.php?cod_producto=956

2. Ciapponi A. Nueva guía de cuidados preventivos de la Fuerza de Tareas de Estados Unidos (primera entrega). Evid actual pract ambul 2005;8:179-182. Disponible en: http://www.foroaps.org/files/nueva\%20fuerza.pdf

3. Adaptado por Ciapponi A de la. Nueva guía de cuidados preventivos de la Fuerza de Tareas de Estados Unidos (segunda entrega). Evid. actual. práct. ambul; 9(1): 26-30, Ene-Feb 2006. Disponible en: http://www.foroaps.org/files/cuida\%20preven\%202\%20parte.pdf 